\title{
Resistance of Mycobacterium Tuberculosis to First and Second Line Anti Tuberculosis Drugs in South West, Nigeria
}

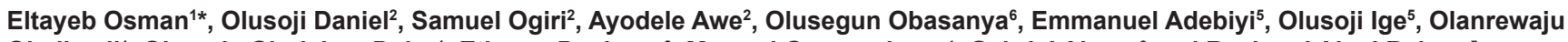
Oladimeji ${ }^{1}$, Olapade Gbolahan Dairo ${ }^{1}$, Etienne Declercq ${ }^{3}$, Mourad Gumusoboga ${ }^{4}$, Gabriel Akang ${ }^{6}$ and Rasheed Ajani Bakare ${ }^{5}$

${ }^{1}$ Damien Foundation Belgium (DFB), Nigeria

${ }^{2}$ World Health Organisation (WHO), Nigeria

${ }^{3}$ Damien Foundation Belgium (DFB), Belgium

${ }^{4}$ Institute of Tropical Medicine Antwerp, Department of Microbilogy, Belgium

${ }^{5}$ University College Hospital Ibadan, Oyo State, Nigeria

${ }^{6}$ National Tuberculosis and Leprosy Control Programme, Nigeria

\section{Abstract}

Setting: Tuberculosis treatment centers in Oyo and Osun States, Nigeria

Objective: This study was aimed to determine the proportion and resistance pattern of Mycobacterium tuberculosis isolates among category 2 failures in Nigeria.

Design: This is a retrospective study of TB Category 2 failures from Oyo and Osun states (Nigeria), from July 2007 to December 2010. Sputum and culture growth of Pulmonary Tuberculosis (PTB) patients were tested for Drug Susceptibility Testing (DST) to first and second line anti-TB drugs in the supra national laboratory at Antwerp, Belgium.

Results: Of the 82 patients, acquired resistance to all first line drugs (RHE and S), were 31(37.8\%), while MDR was observed in $46(56.1 \%)$ of the patients under the study.

Conclusion: MDR TB was high (56.1\%) among patients previously treated with anti-TB drugs, however XDR TB was not observed in the study population. DST services should be made available and accessible to all MDR-TB suspects in the country.

Keywords: MDR; Tuberculosis; Drug suseptabilty testing; Resistance; Nigeria

\section{Introduction}

Tuberculosis (TB) is a major public health problem in Nigeria, being one of the ten leading causes of hospital admissions, [1] and a leading cause of death in adults, especially among the economically productive age group. Nigeria ranks number four in World Health Organisations list of 22 high burden countries worldwide that account for about $80 \%$ of the estimated number of TB cases globally and first in Africa [2]. The emergence of anti tuberculosis drug resistance is an increasing public health problem and TB control programmes in industrialised and developing countries alike [3]. The magnitude of TB in the Nigeria is unknown because population based TB prevalence survey is yet to be conducted, but according to WHO estimates for 2007, the country has an estimated 772,000 cases of all forms of TB, estimated prevalence of $521 / 100,000$ and 195,000 new smear positive cases. By using a population census figure of $148,093,000$ for 2007 , WHO estimates an incidence rate (all cases) of 311 per 100,000 population /year, and for new smear positive, an incidence rate of 131 per 100,000 population/ year [4].

There is currently no country wide drug resistance survey and that is why the exact data about the prevalence and pattern of drug-resistant strains of M. tuberculosis is unknown [5]. However WHO estimates that $1.8 \%$ of all new TB cases and $9.4 \%$ of all re-treatment cases in the country have MDR-TB. There are therefore between 5,000 and 9,000 MDR-TB cases in Nigeria [4].

This study was undertaken to determine the prevalence of $M$. Tuberculosis isolates to first and second line anti TB drugs, determine the existence and burden of MDR/XDR among category 2 failures in two states namely Oyo and Osun states in South West geopolitical zone of Nigeria.

\section{Materials and Methods}

This is a retrospective study in which we analysed the prevalence of drug resistance in $M$. tuberculosis isolated from sputum samples of patients' attending various treatment centres in two states (Oyo and Osun) in Nigeria (supported by Damien Foundation Belgium) from April 2007 - December 2010.

Under the National TB treatment guidelines in Nigeria, there are two categories of treatment which are:

\section{Category 1 treatment}

Patient in this category are initially treated with first line anti TB drugs namely fixed drug combination of Rifampicin, Isoniazid, Ethambutol and Parazinamide for 2 months on intensive phase and commenced on continuation phase with fixed drug combination of Ethambutol and Isoniazid for 6 months. This category of treated are for new TB cases who have never been exposed to anti TB drugs before or for less than 4 weeks

\section{Category 2 treatment}

This category of treatment is reserved for previously treated patients such as patients who failed category 1 treatment (treatment failure) or for patients who returned to treatment having previously defaulted category 1 treatment (return after default), or patients previously declared cured or treatment completed with category 1 regimen

*Corresponding author: Dr. Eltayeb Osman, Damien foundation Belgium, Nigeria Tel: +234-8023683356; E-mail: osman_eltayeb@hotmail.com

Received November 29, 2011; Accepted April 21, 2012; Published April 23, 2012

Citation: Osman E, Daniel O, Ogiri S, Awe A, Obasanya O, et al. (2012) Resistance of Mycobacterium Tuberculosis to First and Second Line Anti Tuberculosis Drugs in South West, Nigeria. J Pulmon Resp Med S6:001. doi:10.4172/2161-105X.S6001

Copyright: (C) 2012 Osman E, et al. This is an open-access article distributed under the terms of the Creative Commons Attribution License, which permits unrestricted use, distribution, and reproduction in any medium, provided the original author and source are credited. 
and are diagnosed with smear positive TB (relapse). The category 2 treatment includes the addition of streptomycin for 2 months together with fixed drug combination of Rifampicin, Isoniazid, Ethambutol and Pyrazinamide for 3 months. The continuation phase uses fixed drug combination of Rifampicin, Isoniazid, and Ethambutol for 5 months.

The patients included in this study were Category 2 failure patients, who never converted while on treatment or who become sputum AFB positive at 5 or 7 months.

\section{Sample collection}

Three sputum samples were obtained from each patient for routine diagnostic purpose in the field, but for the study purpose we obtained another two sputum specimens from the positive ones.

Two types of specimens were sent to the Supranational Laboratory at Antwerp Institute of Tropical Medicine in Belgium, sputum samples and colonies from culture growth.

For sputum; two sputum specimens from each patient were collected and kept in 50mL screw cap centrifuge tube (falcon). 5-7 mL of sputum with an equal volume of $\mathrm{CPC}-\mathrm{NaCl}$ was added to the sputum. The containers were securely capped and shacked by hand until specimens are liquefied. The caps are properly sealed and specimens were sent to Antwerp. The average period from collection to be delivered to the lab is 8 days, express couriers were used.

For the culture: sputum was first cultured at Ibadan University Hospital College (UCH) Laboratory using Ogawa media method for Mycobacteria isolation, and after the growth of the bacteria we shipped the specimens to Antwerp using sterile $2 \mathrm{~mL}$ cryovials.

Two cryovials were prepared per strains; in one of cryovial $1 \mathrm{~mL}$ of $1 \%$ CPC was added. The harvested colonies are transferred into the $2 \mathrm{~mL}$ cryovial containing $1 \mathrm{~mL}$ of CPC $1 \%, \mathrm{NaCl} 2 \%$. In the second one, the harvested colonies were transferred directly, without any preservative component (dry colonies). Results of the sample isolates were received on average of about 4 months after the samples were sent.

Details of respondents about socio-demographic characteristics and previous treatment history of the patients were obtained from their treatment cards and TB registers at their respective treatment centers. Data was initially entered into an excel sheet and analysis was done using EPI Info 2002.

\section{Results}

A total of 82 patients who had failed category 2 treatment under the national TB treatment guidelines were included in this study. There were 49 males $(59.8 \%)$ and 33 females $(40.2 \%)$. The age range was 20 60 years. The mean age was $36.0 \pm 9.1 \mathrm{yrs}$, while the peak age group was $30-39(50.0 \%)$ years as shown in Table 1 . There was no significant difference in the mean age between males and females $(37.1 \pm 9.1$ vs $34.3 \pm 8.9 ; \mathrm{p}=0.18$ ).

\begin{tabular}{|l|c|c|c|}
\hline Age(yrs) & Female $\mathbf{( n = 3 2 )} \mathbf{n}(\mathbf{\%})$ & Male $(\mathbf{n}=\mathbf{4 8}) \mathbf{n}(\mathbf{\%})$ & Total $\mathbf{( N = 8 0 )} \mathbf{n}(\mathbf{\%})$ \\
\hline $20-29$ & 9 & 9 & $18(22.5)$ \\
\hline $30-39$ & 15 & 25 & $40(50.0)$ \\
\hline $40-49$ & 4 & 5 & $9(11.3)$ \\
\hline $50-59$ & 4 & 8 & $12(15)$ \\
\hline$\geq 60$ & 0 & 1 & $1(1.2)$ \\
\hline Total & $32(40)$ & $48(60)$ & $80(100)$ \\
\hline
\end{tabular}

The ages of two respondents were missing (one male and one female)

Table 1: Age and Sex Distribution of PTB patients in Oyo and Osun States.

\begin{tabular}{|l|c|}
\hline Resistance & Frequency $\mathbf{n}(\%)$ \\
\hline $\mathbf{H}$ & $52(63.4)$ \\
\hline $\mathbf{R}$ & $49(59.8)$ \\
\hline $\mathbf{E}$ & $37(45.1)$ \\
\hline $\mathbf{S}$ & $50(61.0)$ \\
\hline HR (MDR*) & $46(56.1)$ \\
\hline HE & $35(42.7)$ \\
\hline HS & $45(54.9)$ \\
\hline RS & $44(53.7)$ \\
\hline RE & $34(41.5)$ \\
\hline ES & $33(40.2)$ \\
\hline HES & $31(37.8)$ \\
\hline RES & $32(39.0)$ \\
\hline HRE & $33(40.2)$ \\
\hline HRS & $42(51.2)$ \\
\hline HRES & $31(37.8)$ \\
\hline
\end{tabular}

*MDR, defined as resistance to at least $\mathrm{H}$ and $\mathrm{R}$

$\mathrm{H}=$ Isoniazid, $\mathrm{R}=$ Rifampicin, $\mathrm{E}=$ Ethambutol, $\mathrm{S}=$ streptomycin, $\mathrm{Z}=$ Pyrazinamide (not tested)

Table 2: Drug resistance patterns to first line anti-TB drugs of Mycobacterium tuberculosis isolated from TB patients in Oyo \& Osun States $(n=82)$.

\begin{tabular}{|l|c|}
\hline Resistance & Frequency $\mathbf{n ~ ( \% ) ~}$ \\
\hline O & $7(8.5)$ \\
\hline P & $3(3.7)$ \\
\hline ET & $6(7.3)$ \\
\hline K & $2(2.4)$ \\
\hline C & $2(2.4)$ \\
\hline
\end{tabular}

$\mathrm{K}=$ kanamycin, $\mathrm{C}=$ cyclserine $(\mathrm{c}), \mathrm{O}=$ ofloxacin, $\mathrm{P}=$ prothinamide, $\mathrm{ET}=$ ethionamide Table 3: Drug resistance patterns to second line drugs of Mycobacterium tuberculosis isolated from TB patients in Oyo \& Osun States $(n=82)$.

The resistance pattern of the M.TB isolates to first line anti-TB drugs among category 2 failures were described in Table 2. MDR TB (resistance to Rifampicin and Isoniazid) was detected in 46(56.1\%) of the patients. There was no statistical difference in the age of patients diagnosed as MDR-TB and non MDR-TB patients ( $35.5 \pm 8.7$ vs $36.6 \pm$ 9.6; $\mathrm{p}=0.6)$. Overall acquired resistance to all the first line drugs RHE and $\mathrm{S}$ was $31(37.8 \%)$.

\section{Second line drug resistance}

All the isolates from the 82 patients were subjected to drug susceptibility testing to second line drugs, kanamycin (K), cyclserine(c), ofloxacin $(\mathrm{O})$, prothinamide $(\mathrm{P})$ and ethioamide $(\mathrm{ET})$.

Six (7.3\%) patients had resistance strain to ethioamide (ET), 7 (8.5\%) to ofloxacin $(\mathrm{O}), 3(3.7 \%)$ to prothinamide $(\mathrm{P}), 2(2.4 \%)$ to kanamycin and $2(2.4 \%)$ to cyclserine(c) as shown in Table 3.

Among the MDR isolates 6 patients had resistance strain to ofloxacin (13.0\%), 4 to ethionamide (8.7\%) and 3 to prothionamide (6.5\%). One patient showed resistance to the 4 first line drugs (RHES) in addition to both ofloxacin and ethionamide (RHESET), and one patient had resistance to ethionamide and kanamycin in addition to the 4 first line drugs (HRES). No case of XDR-TB was diagnosed in the study as shown in Table 4.

\section{Discussion}

The study is an outcome of retrospective analysis of data collected from 2007- 2010 on drug resistance profile of M. tuberculosis from patients diagnosed with pulmonary TB by smear microscopy. The results may be considered to be representative for the rural population 


\begin{tabular}{|l|c|}
\hline Resistance & $\mathbf{n}(\%)$ \\
\hline RHK & $2(4.3)$ \\
\hline RH(ET) & $4(8.7)$ \\
\hline RHO & $6(13.0)$ \\
\hline RHP & $3(6.5)$ \\
\hline RH(ET)K & $1(2.1)$ \\
\hline RHO(ET) & $1(2.1)$ \\
\hline RHOK & 0 \\
\hline RHOC & 0 \\
\hline RHOP & 0 \\
\hline
\end{tabular}

$\mathrm{H}=$ =Isoniazid, $\mathrm{R}=$ rifampicin, $\mathrm{K}=$ kanamycin, $\mathrm{C}=$ cyclserine $(\mathrm{c}), \mathrm{O}=$ ofloxacin, $\mathrm{P}=$ prothinamide, ET= ethionamide

Table 4: Drug resistance patterns of MDR patients to Second line drugs $(n=46)$.

of Oyo \& Osun States, but should not be extrapolated to other areas, and certainly not urban ones.

In the present study the resistant rates were highest for Isoniazid (63.4\%), followed by streptomycin (61.0\%), by Rifampicin (59.8\%) and the least ethambutol (45.1\%). Among the study population the overall MDR rate is high (56.1\%). This result shows high rate of MDR compared to Abuja study that detected $4 \%$ of overall MDR [6] but lower than $87 \%$ among category 2 failures patients observed in a study in Bangladesh [7]. The high MDR rate in this study may not be unconnected to the selection bias of patients who had failed previous treatment.

The findings in this study are similar to the study in Bangladesh which showed similar high rates to INH and Rifampicin but a much lower rate to ethambutol [7]. The high resistance to streptomycin may be due to the fact that the drug is generally used for the treatment of other clinical conditions and is available in the open market. If this drug will still be relevant in the treatment of category 2 patients then there might be a need to control the use of the drug in the country.

As at the time of the study, second line anti TB drugs were not available in the country and as such patients diagnosed are not under standardized or individualized category 4 regimen. In many instances some physicians in a bid to offer some palliative measures add a fluoroquinolone which are readily available in the open market, to the failing or failed regimen. This action invariably provides a good environment for the development of XDR TB in such patients.

With the high proportion of MDR TB in this study and without appropriate treatment, there is high morbidity and mortality among these patients with the possibility of continued transmission with the community where proper infection control measures are not instituted. There is need for the national programme to set in motion the mechanism to set up guidelines and train medical practitioners in the clinical management of these patients and in addition procure second line drugs to patients who need them.

Currently a national MDR survey is being carried out to know the burden and the pattern of drug resistance in the country. This will assist the national government to have a baseline that will assist in planning, monitoring and the establishment of a surveillance system to monitor the trend of MDR in the country.

The need to strengthen the routine DOTS cannot be overemphasized especially in resource limited countries where there is resource constraint to purchase second line drugs that are very expensive and not readily available. There is need to also prevent the development of drug resistant $\mathrm{TB}$ by ensuring that all TB patients whether in private or public facilities are treated strict compliance to the national TB guidelines regarding chemotherapy regimens and case holding methods, so as to ensure that all cases of TB are treated correctly.

No XDR TB was observed in this study, though there were some resistant strains to some second line drugs. This situation can only be maintained where the second line drugs are controlled and not available in the open market. In addition medical practitioners need to be properly trained on the rational use of second line drugs. With the very high mortality associated with XDR TB as reported in some countries, Nigeria cannot afford to create XDR TB situation as a result of the mismanagement of MDR TB. There is need to develop and widely disseminate the MDR TB guidelines and protocols and the need for all partners to support the process.

There is need for the national programme to develop a national drug resistance surveillance system to monitor resistance rates among different categories of TB patients which can provide useful indications as to what chemotherapy regimens to adopt in the case management of MDR-TB patients.

\section{Conclusion}

This is the only study conducted in Nigeria under a supranational laboratory to determine the prevalence and the pattern of drug resistance in the country, in the absence of any national data about the prevalence and incidence of multi-drug-resistant strains of $M$ tuberculosis.

Our findings are of concern and raised an alarm about the existence of drug resistance in the country and if no effective measures be undertaken it will become a major public health problem.

The findings suggest that the cases of MDR TB in Oyo \& Osun states may have appeared for any of 2 reasons: endogenous development of MDR strains due to inadequate case management, or ongoing transmission.

This study points to an urgent need for the establishment of facility for external quality assured DST in different parts of the country to accurately determine the pattern and extent of drug resistance TB and the magnitude of MDR in the country.

The first priority is full coverage and encouragement of private sectors physicians to apply correct regimens following the National TB \& Leprosy Control Programme guidelines, and treatment must be under close supervision.

The enhancement of infection control measures to prevent transmission, the expansion of high quality diagnostic services for timely detection of cases, and community involvement to improve adherence are three priority areas that need more attention, but perhaps most importantly, the development of treatment programmes into which patients can be enrolled and treated successfully.

\section{Acknowledgement}

We are thankful to the Institute of Tropical Medicine at Antwerp and $\mathrm{UCH}$ laboratory personnel.

We thank the State TB officers', TB supervisors in Oyo and Osun States. This work was supported by the Damien Foundation Belgium

Source of support. Damien Foundation Belgium.

\section{References}

1. Busari OA, Olarewaju OT, Busari OE (2009) Management and outcomes of suspected pulmonary tuberculosis in a resource-poor setting, The Internet Journal of Pulmonary Medicine 11: 1-5.

2. World Health Organisation (2011) Global Tuberculosis Control: a short update to 2009 report. 
Citation: Osman E, Daniel O, Ogiri S, Awe A, Obasanya O, et al. (2012) Resistance of Mycobacterium Tuberculosis to First and Second Line Anti Tuberculosis Drugs in South West, Nigeria. J Pulmon Resp Med S6:001. doi:10.4172/2161-105X.S6-001

3. World Health Organisation (2008) World TB Day 2009, Anti Tuberculosis Drug Resistance in the World, Fourth Global Report. The WHO/IUATLD Global Project on Anti-tuberculosis, Drug Resistance Surveillance, 2002-2007.

4. World Health Organization (2008) Global tuberculosis control. WHO/HTM/ TB/2008, 393.

5. World TB Day 2009 WHO calls for implementation of the 'STOP TB' strategy in Nigeria.
6. Osagie EADK, Shuaib A, Lawson L (2008) Multidrug-resistant tuberculosis at the National Hospital, Abuja, Nigeria. African Journal of Respiratory Medicine 23.

7. Rahman M, Kamal SM M, Mohammed FR, Alam B Md, Ahasan HAM N (2009) Anti tuberculosis drug resistance pattern among different category of tuberculosis patients. J Medicine 10: 45-47. 